\title{
STUDY OF CAD-INTEGRATED ANALYSIS FOR COMPLEX STRUCTURES
}

\author{
Hirpa L. Gelgele \\ Dept. of Mechanical/Structural Engineering and Material Technology, \\ University of Stavanger, Norway \\ Email: Hirpa.G.Lemu@uis.no
}

\begin{abstract}
In the classical product development process, design of a product is verified by testing physical prototypes. This approach falls short of fulfilling the demands for providing products at lower cost and short delivery time. This article illustrates the use of the digital data from CAD model in analysis and simulation of complex structures. Two industrial examples are shortly discussed to demonstrate the advantages of integrating analysis in the early phase of the product development process.
\end{abstract}

Key words: Product development, finite element analysis, integrated CAD/FEA, CAE

\section{INTRODUCTION}

Providing products to the market at lower prices, shorter time and better quality have been the means of gaining better competitive advantage for manufacturing industries within the last $4-5$ decades. For products that require mechanical design, in particular, being able to use computers in the design process was the first step towards design automation that resulted in today's CAD (Computer-Aided Design) systems. For example, the early computer aided drafting system like CADAM from Lockheed Martin were developed to reduce time to market by replacing the conventional drawing board in early 1970s. Manufacturing engineers have also attempted to automate the machining process through developing numerically controlled tools such as APT (Automated Programming Tool) from MIT. However, the standalone Computer-aided technologies faced short of fulfilling the required goal due to constraints of lack of common language between the CA-techniques. Research in late $80 \mathrm{~s}$ and early $90 \mathrm{~s}$ has focused on

Please use the following format when citing this chapter:

Gelgele, Hirpa, L., 2006, in International Federation for Information Processing (IFIP), Volume 207, Knowledge Enterprise: Intelligent Strategies In Product Design, Manufacturing, and Management, eds. K. Wang, Kovacs G., Wozny M., Fang M., (Boston: Springer), pp. 673-678. 
establishing an integrated system between CAD/CAM systems including the automation of process planning, production control and the like.

In the traditional design methodology, verification of the design process is based on testing physical prototypes that have similar performance to the required product or machine with certain dimensional scaling. For complex machines and structures, however, this approach is not only difficult, but also time and resource consuming.

This article illustrates an integrated approach to early analysis of complex products at the design phase using Finite Element Analysis (FEA) as a replacement for simulations and verifications using physical prototypes.

\section{WHY ANALYSIS AT EARLY DESIGN PHASE?}

As stated above, verification of the design for complex products through prototype testing is a time-consuming process. Studies show that $85 \%$ of the total time and cost of product development are committed in the early stages of the product development phase when only $5 \%$ of the project resources are expended. This is because in the early conceptual stage, necessary decisions are made concerning basic geometry, material type, system configuration and manufacturing processes. Further, mistakes or wrong decisions made at the early stage of product development phase can be modified with none or minimal increment in the product cost. In other words, the time and cost to correct problems increases about ten-fold with each step of the product development cycle: concept definition, detailed design, prototype development, prototype testing, and manufacturing of final product.

Industries have attempted to improve these and other drawbacks of the traditional product development process through extensive use of computers in various phases of the process, concurrent engineering, computer integrated design and manufacturing systems, and other product development compression techniques. These methods have shown better results for products of few components, simpler geometry and configuration.

\section{AN INTERACTIVE DESIGN AND SIMULATION}

Today, several computer-based tools have been available to assist a product's life cycle. Solid modeling has enabled designers to define parts and assemblies in 3D space and utilize the geometric data in several of downstream applications such as process planning, prototyping, simulation and analysis. It has been possible to convert CAD models into physical prototype parts in order to visualize, fit together and evaluate the appearance 
of components. Using recently developed knowledge-based systems; we can capture technical standards, experiential know-how, defined procedures and other design and manufacturing information to automate design routines.

Most recently, the term Computer-aided engineering (CAE) appeared in the engineering field to imply the interactive work of CAD, CAM and FEA (Finite Element Analysis) systems. This concatenation of FEA in $\mathrm{CAD} / \mathrm{CAM}$ systems to give birth to CAE systems indicates the necessity to study stress, deformation, vibration, temperature distribution and other behavior of products or structures using the CAD data. As far as these tools are computer-based, being able to share the digitalized information among these tools truly contributes to the compression of the product's time to market.

\subsection{Existing implementation challenges}

The primary precondition for an effective application of numerical analysis tool in engineering to detect potential areas having excessive stress, deformation, vibration and/or other problems in complex structures is existence of a 3D model. An interactive design and analysis through integration of CAD and FEA tools allows designers to quickly iterate the proposed design solution and evaluate the merits and demerits of different ideas, compare alternatives, and identify design weaknesses before a costly production has been done.

However, the real advantages of this tool cannot be achieved without problems. Primarily, the powerful graphics capabilities, and specially the non-standardized user interfaces require considerable time and expertise of a dedicated analyst having the basic knowledge of finite element methods (FEM) and FEA theory. As FEA is an approximation method, the level of the analysis error depends on the skill, training and background of the user to apply proper mesh densities, element types and boundary conditions. Furthermore, the analyst must have sufficient knowledge to translate CAD geometry into proper format for building the FEA model, as well as appropriately interpret results, plots and other output information.

In many manufacturing companies, this knowledge gap between the designer and the analyst is so high that FEA is typically reserved for critical components. To have a competitive advantage, however, efforts are being done to re-orient the product development process itself so that analysis is performed much earlier in product development [Roth, 2000].

The other issue is that the embedded analysis systems within the CAD environment often do not contain the full capabilities that a standalone FEA system would have, and this has to be addressed in future developments. 


\subsection{FEA can replace physical testing}

The trend in the manufacturing industry shows that the cost of materials and manpower to build prototypes is ever increasing. Conversely, computer hardware costs decreased significantly and computing power is increasing, which has led to a wider use of virtual prototyping using CAE tools.

Application of FEA on solid models of products benefits manufacturers in that they don't rely on expensive and time-consuming prototypes. While physical tests can still be required for certain product certifications, simulation using 3D models is more cost-effective and repeatable than physical tests. The other advantage of integrating FEA in early design phase is that it reduces the knowledge gap by allowing the test and redesign performed on software prototypes than on physical prototypes (Adams and Askenazi, 1999).

\subsection{Industrial examples}

\subsubsection{Analysis of a robot arm}

In this section, the rationale for virtual prototyping at the early product development phase or as an integrated part of the whole design process for an industrial robot is illustrated. The studied robot has motion about 6 axes. The load in the components is not so high but very complex during the motion in those different axes.
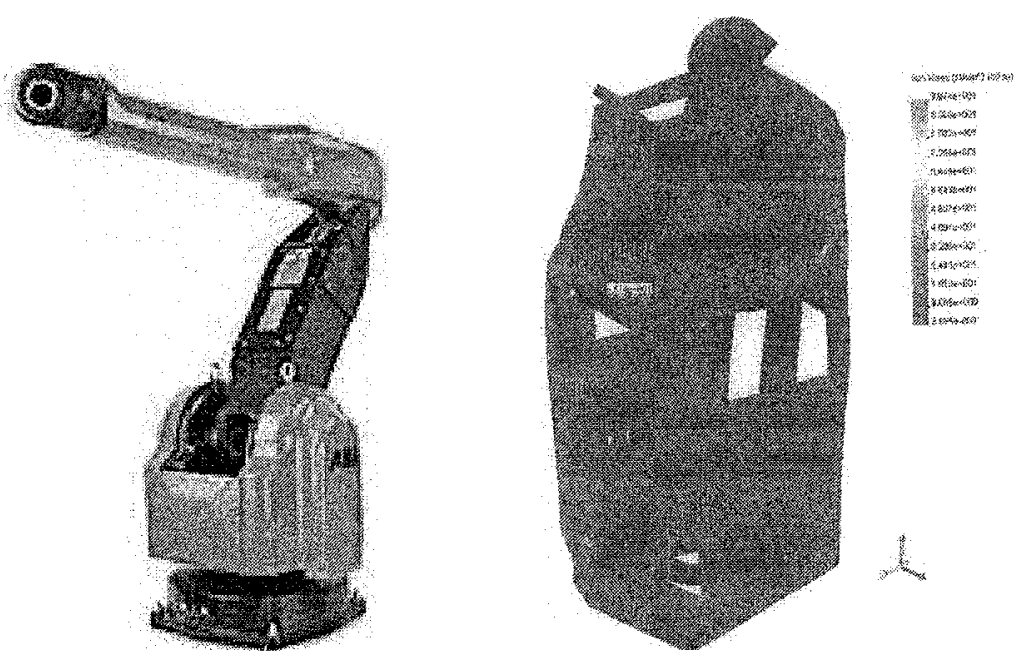

Figure 1. Analysis of robot arm 
Because of the precision required, robots are traditionally designed for high stiffness. On the other hand, the vertical arm of the robot is exposed to various forms of loading, and at the same time stress concentration due to access holes for electronic components makes this part of the robot the weakest link. This weak part also influences the programmed precision. The robot arm has two parts:

- Upper part - of Aluminum alloy AlSi10Mg, yield strength $200 \mathrm{MPa}$ and

- Lower part - of nodular graphite iron SjK-400, yield strength $250 \mathrm{MPa}$ (analysis model not shown).

The modeling was done in SolidWorks, a 3D CAD software. The fully embedded analysis software COSMOSworks within the 3D CAD environment facilitates the smooth transfer of the geometry to the analysis model. Based on the geometry data, the analysis model describes the product in terms of finite element meshes, loads and constraints. Using this approach, it has been possible to make many "what-if" scenarios and identify the weakest portion of the structure for various loading and motions while it is still in digital form.

Nowadays, many manufacturing industries are re-orienting their design procedures so that a single digital file (i.e. the solid model) contains all necessary information of the product's life cycle. Studies in the local industry show also that virtual analysis at early phase of the product development process has, among others, the following advantages:

- Reduced product development time $40-50 \%$

- Reduced design error $30-35 \%$

- Improved customer satisfaction

- Simplified product configuration

- Improved product quality and safety.

\subsubsection{Stress concentration factor in a pressure vessel}

The effect of stress concentration in pressure vessels is mostly estimated using hand calculations based on empirical relations. The results of such calculations are very unreliable particularly in the localized high stress areas due to holes, welded joints and dimensional variations. Studies (Sang et al., 2002) show that a reliable and accurate design method for pressure vessel intersections with diameter ratio $d / D \geq 0,5$ is necessary, where $d=$ nozzle diameter and $\mathrm{D}=$ shell diameter.

In this study, a cylindrical pressure vessel with spherical ends and mean diameter $\mathrm{D}=800 \mathrm{~mm}$ was modeled in the CAE tool I-DEAS $10 \mathrm{NX}$ series. The cylindrical shell with a cylindrical nozzle at the middle is positioned horizontally and supported at two bottom points. 

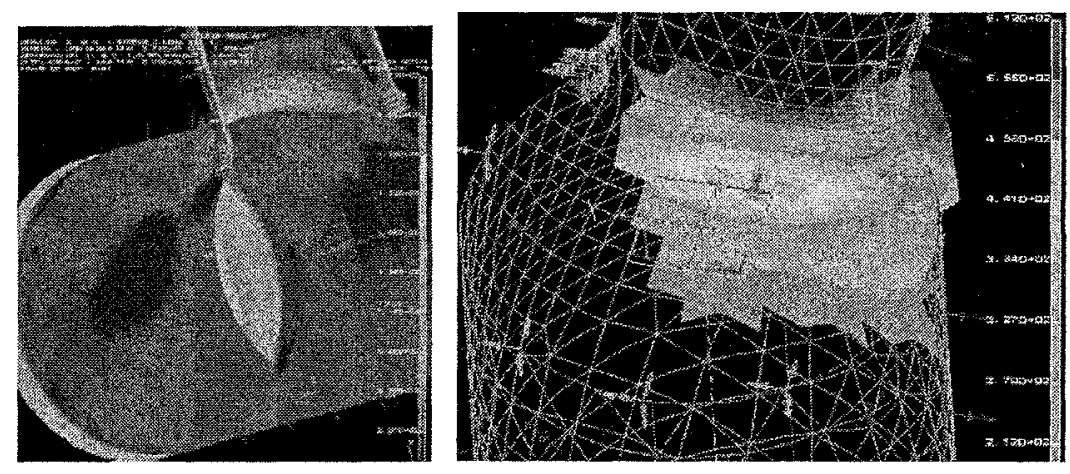

Figure 2. Analysis of stress concentration factor in a pressure vessel

The main objective of this study is to identify how local stresses vary with dimensions of a pressure vessel, where making physical tests are not amenable due to certain practical reasons. The results show that the stress concentration factor for a given $\mathrm{D} / \mathrm{d}$ ratio decreases with increasing fillet radius (Gelgele, 2005). At the same time it has been observed that smaller $\mathrm{D} / \mathrm{d}$ ratio has higher stress concentration factor.

\section{CONCLUSIONS}

The article demonstrated the possibilities and advantages of executing analysis of complex structures at an early design phase. An integrated CAD/FEA approach enables to study the performance of structures by varying design parameters in a cost effective way using the digital data of the CAD model. This signifies the transition from the traditional design by formula to design by analysis approach. However, there are still many practical issues that have to be addressed to get the real implementation.

\section{REFERENCES}

Adama, V. and Askenazi, A. (1999), Building better products with Finite Element Analysis, OnWord Press, 1 . ed.

Geigele, H. L. (2005), Stress concentration in pressure vessels using FEM, Proc. of $9^{\text {th }}$ Int. conf. on Trends in development of machinery and related technologies, Antalya, Turkey.

Roth, G. (2000), The value of early analysis, Benchmark, Int. magazine for engineering design and analysis, NAFEMS.

Sang, Z. F. at el., (2002) Limit and burst pressures for cylindrical shell intersection with intermediate diameter ratio, Int. Journal of Pressure Vessels and Piping, 79, 342-349. 